\title{
CVD diamond wires and tubes
}

\author{
P. W. May, C. A. Rego, R. M. Thomas, M. N. R. Ashfold and K. N. Rosser \\ School of Chemistry, University of Bristol, Cantock's Close, Bristol BS8 1TS (UK)
}

N. M. Everitt

Department of Aerospace Engineering, University of Bristol, University Walk, Bristol BS8 1TR (UK)

\begin{abstract}
Diamond has been uniformly deposited onto the surface of thin metal wires using hot filament CVD. The diamond-coated wires are stronger and stiffer than the uncoated wires. Subsequent etching of the metal core in a suitable chemical reagent allows free-standing diamond tubes to be made, the typical dimensions being $1 \mathrm{~cm}$ long with an internal diameter of 10-150 $\mu \mathrm{m}$. The formation of a thick, chemical-resistant carbide layer at the metal-diamond interface when using $\mathrm{Ti}$ and $\mathrm{W}$ wires is investigated.
\end{abstract}

\section{Introduction}

The discovery of a means to produce thin films of diamond by chemical vapour deposition (CVD) $[1,2]$ has opened the door to many potential applications, including optical components, semiconducting devices and hard, wear-resistant coatings [3, 4]. To date, most reports of CVD diamond have been restricted to planar substrates, usually of materials such as $\mathrm{Si}$ or Mo. A whole new set of potential applications becomes possible, however, if CVD diamond can be grown onto nonplanar substrates.

In previous reports, ourselves and other workers, have demonstrated the ability to grow diamond films uniformly on the surface of metal wires [5-8]. By subsequent removal of the metal core, free-standing diamond tubes, or hollow fibres, may also be fabricated. However, these reports only give initial findings and lack detailed accounts of the important experimental considerations. Therefore, in this paper, we examine in more detail the effect upon diamond deposition of the composition of the wire substrate, with particular reference to carbideforming metals such as $\mathrm{W}$ and $\mathrm{Ti}$.

\section{Experimental details}

Diamond deposition was performed in a modified hot filament reactor, operating under standard CVD growth conditions [5]. A gas mixture of $1 \%$ methane in $\mathrm{H}_{2}$ was admitted to the reactor at a total flow rate of $200 \mathrm{sccm}$ (standard cubic centimetres per minute) and a pressure of 30 Torr. A Ta filament at a temperature of $2000{ }^{\circ} \mathrm{C}$ was used to activate the gas mixture, causing diamond to deposit onto a wire substrate. The deposition rate was about $0.5 \mu \mathrm{m} \mathrm{h}^{-1}$.

To ensure uniform coating around the wire, the individual wires were positioned centrally and coaxially within the coils of the filament [5]. This represents an advance over previously reported deposition geometries, where the wire was mounted (as for a planar substrate) below or beside the filament. The present design offers the possibility of uniformly coating larger diameter wires (up to a few millimetres) simply by enlarging the filament coil to maintain the filament-substrate distance at the optimum value ( $4-5 \mathrm{~mm}$ in our reactor) for the growth of good quality diamond films. In this design, the wires are mounted vertically, and are weighted to keep them straight and to prevent them warping during deposition. The surface temperature of the wire during growth was not measured, owing to the difficulty of placing a thermocouple through the filament. However, comparing the growth rate of diamond on planar substrates with that found on wires, we estimate the wire surface temperature to be around $900{ }^{\circ} \mathrm{C}$. The proximity of the filament enabled this substrate temperature to be maintained without an additional substrate heater being needed.

Metal wires were made from $\mathrm{Cu}, \mathrm{Ti}$ and $\mathrm{W}$, and ranged in thickness from 10 to $350 \mu \mathrm{m}$ and in length from 1 to $2 \mathrm{~cm}$. The wires were manually abraded with 1-3 $\mu \mathrm{m}$ diamond grit prior to deposition. Results for diamond-coating of other metal wires, such as Mo or $\mathrm{Ta}$, and non-metal fibres, such as silicon carbide or silica, are presented elsewhere $[5,6,9]$. Laser Raman spectroscopy was used to characterize the deposited layers and invariably gave a strong $1332 \mathrm{~cm}^{-1}$ peak, characteristic of diamond, with very little contribution from non-diamond phases at $1550 \mathrm{~cm}^{-1}$. Scanning 
Auger spectroscopy was used to determine the composition of the interface layers and to provide unambiguous identification of the carbide layers. A more detailed investigation of the interfacial properties of CVD diamond coatings on wires is currently under way [10], and will be presented in future publications.

After deposition, the metal core was etched away using a suitable chemical reagent, leaving a free-standing diamond tube of typical length $5 \mathrm{~mm}$. The $\mathrm{Cu}$ was etched in concentrated nitric acid, the $\mathrm{W}$ in hot hydrogen peroxide solution and the $\mathrm{Ti}$ in either $\mathrm{HF}$ solution or a solution prepared by dissolving $\mathrm{NaF}$ in concentrated $\mathrm{HCl}$.

\section{Results}

The results are presented for CVD of diamond onto wires of three different metals, in turn, along with the outcome of the subsequent acid etching studies to produce hollow diamond fibres.

\subsection{Copper}

$\mathrm{Cu}$ suffers from two problems with regard to diamond deposition: (i) it does not form a carbide, the presence of which is thought to aid diamond-substrate adhesion; (ii) it has a much higher thermal expansion coefficient than that of diamond. This means that, on cooling from the deposition temperature, the $\mathrm{Cu}$ wire contracts inside its diamond coating, producing compressive stresses within the diamond layer. For $\mathrm{Cu}$ wires more than $100 \mu \mathrm{m}$ in diameter, the stresses were sufficiently large to crack the diamond film, producing flaking and delamination (see Fig. 1). However, diamond-coated $\mathrm{Cu}$ wires of diameter less than $100 \mu \mathrm{m}$ contain a larger volume fraction of diamond for a given thickness of coating. Therefore, thinner wires were less susceptible to cracking

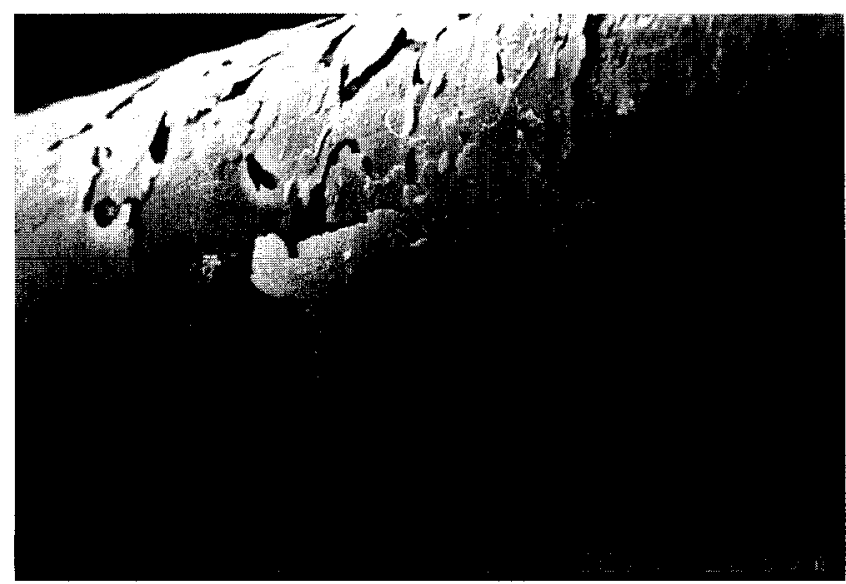

Fig. 1. Diamond film deposited on a $\mathrm{Cu}$ wire $300 \mu \mathrm{m}$ in diameter. The film cracked and flaked during cooling, owing to the expansion mismatch between $\mathrm{Cu}$ and diamond.

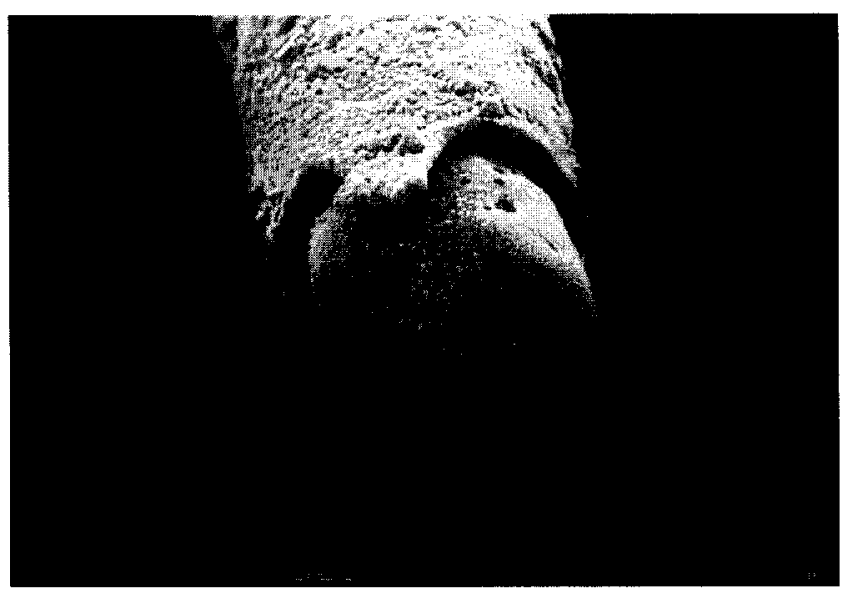

Fig. 2. Diamond layer $5 \mu \mathrm{m}$ thick deposited onto a $\mathrm{Cu}$ wire $50 \mu \mathrm{m}$ in diameter. The increased volume fraction of diamond prevents the diamond film cracking when the $\mathrm{Cu}$ contracts as it cools from the deposition temperature.

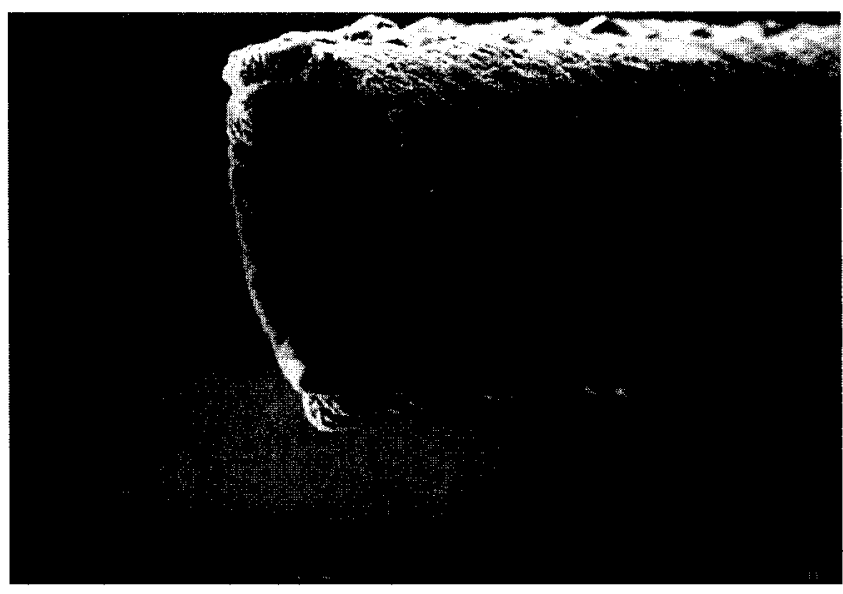

Fig. 3. Free-standing diamond tube, with internal diameter $50 \mu \mathrm{m}$ and wall thickness of $5 \mu \mathrm{m}$, made by depositing diamond on a $\mathrm{Cu}$ wire and then etching away the $\mathrm{Cu}$ in nitric acid.

and could be coated relatively easily with continuous diamond films (see Fig. 2).

The lack of a $\mathrm{Cu}$ carbide layer is actually advantageous, since a simple nitric acid treatment allows complete removal of the metal core, leaving a free-standing diamond tube (see Fig. 3). This is in contrast to the results for strong carbide-forming materials, such as $\mathrm{W}$ and $\mathrm{Ti}$, which leave carbide residues on the inner surface of the tube.

\subsection{Titanium}

Ti also has a large expansion coefficient compared with that of diamond; however, it forms a carbide very easily, which greatly increases the adherence of any diamond film. This allows thin diamond films to be grown on $\mathrm{Ti}$ wires of diameter up to $1 \mathrm{~mm}$ without delamination.

Unfortunately, the carbide layer formed between the 
diamond and $\mathrm{Ti}$ can be very thick (several tens of micrometres [11]) and continues to increase in thickness during diamond deposition. This carbide layer poses a number of problems. First, as $\mathrm{C}$ reacts with the $\mathrm{Ti}$ during deposition, the carbide layer gradually swells inside its diamond jacket. As a result, during long deposition times, the growing diamond coating can burst, resulting in cracks running the length of the wire (see Fig. 4). This places an upper limit on the thickness of a diamond film that can be grown on a $\mathrm{Ti}$ wire.

Etching away the Ti core to produce a diamond tube is also problematical. The $\mathrm{Ti}$ core is easily dissolved using a fluoridized acid etch, but the thick carbide layer is resistant to such chemical attack and remains bonded to the inside walls of the diamond tube. For example, Fig. 5 shows that, after $20 \mathrm{~min}$ of etching, the Ti has been completely dissolved away but the carbide layer still remains. Experiments are presently under way to

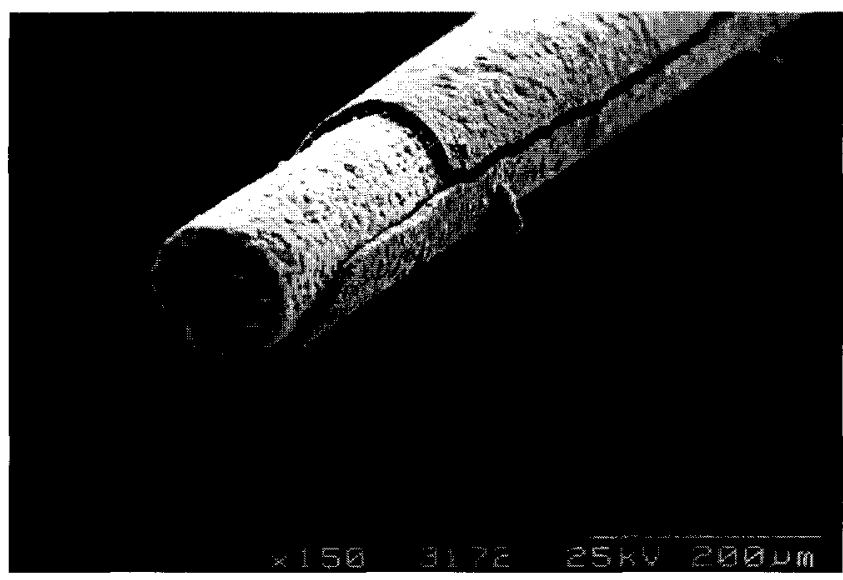

Fig. 4. Longitudinal cracking of the diamond film on a Ti wire $150 \mu \mathrm{m}$ in diameter.

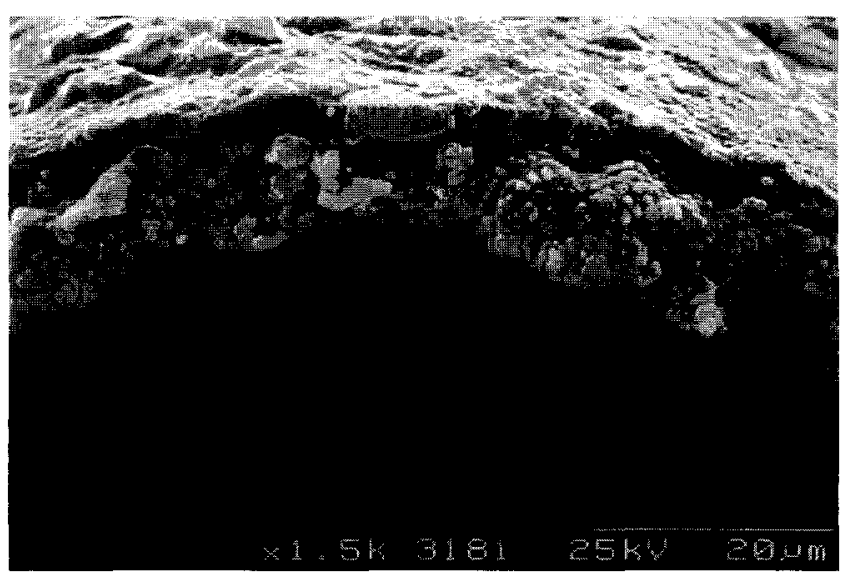

Fig. 5. Micrograph showing part of the tube obtained from a diamondcoated Ti wire $150 \mu \mathrm{m}$ in diameter after $20 \mathrm{~min}$ of acid etching. The $\mathrm{Ti}$ core has completely dissolved but a $\mathrm{Ti}$ carbide layer remains bonded to the inside surface of the diamond. try to identify a suitable chemical reagent to remove this carbide layer without damaging the diamond.

\subsection{Tungsten}

W has a relatively low expansion coefficient, so is a particularly amenable substrate for CVD of diamond (see Fig. 6). It readily forms a carbide, which results in strong adherent $\mathrm{W}$-diamond interfaces, even over quite rugged topologies (Fig. 7). We have demonstrated that continuous diamond films several micrometres thick can be grown on wires of diameter up to $1 \mathrm{~mm}$, without any problems with delamination or cracking of the film. However, tungsten carbide is also resistant to chemical attack and remains bonded to the inner walls of the tubes after etching.

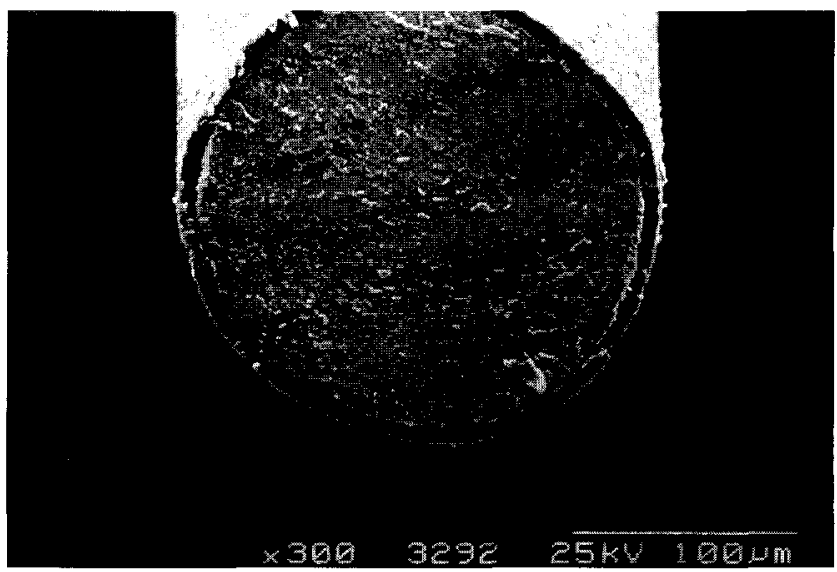

Fig. 6. Micrograph of a diamond-coated $\mathrm{W}$ wire $200 \mu \mathrm{m}$ in diameter, highlighting the uniformity of deposition. The diamond layer is $8 \mu \mathrm{m}$ thick.

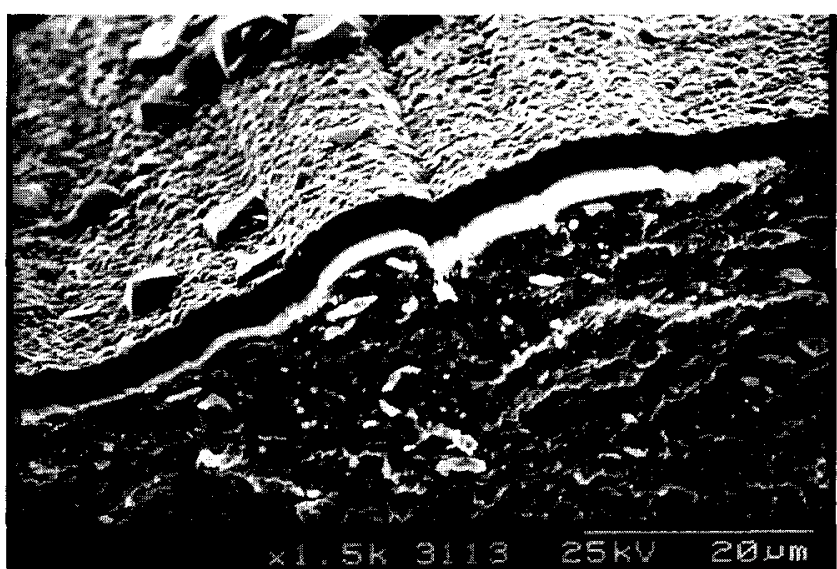

Fig. 7. Micrograph showing the interface of a diamond film grown on a W wire $300 \mu \mathrm{m}$ in diameter. The diamond film closely follows the contours of the wire surface, which were created during manufacturing of the wire. 


\section{Conclusions}

We have demonstrated that it is possible to deposit diamond films onto metal wires and to etch away the cores of the wires to produce hollow diamond fibres. The potential applications of such diamond-coated wires or hollow fibres are diverse. They may find use in applications such as ultrafine hypodermic needles, laser waveguides in optical electronics, or for strengthening metals in the form of fibre-reinforced composites $[6,12]$. The predicted high thermal conductivity for the hollow fibres may be exploited in terms of microchannel cooling, with coolant being pumped through the fibres to allow efficient heat removal from small, high power devices. Hollow tubes also may find uses where stiff supports are required in the absence of metals; for example, in the vicinity of high electric or magnetic fields, or for radar transparent "stealth" applications.

These proposed applications are based upon the predicted properties of diamond fibres, since no results for mechanical testing of similar fibres have yet been reported. However, our preliminary qualitative results indicate that diamond-coated wires are stronger and significantly stiffer than uncoated wires.

The effect upon the physical properties of the hollow fibres of having an inner Ti carbide layer, and whether it is important for any subsequent application, have still to be determined. A suitable chemical etchant for removal of the carbide layer is urgently required.

\section{Acknowledgments}

We would like to thank the DTI for financial support. PWM also thanks the Ramsay Memorial Trust and British Gas for funding. Published with the permission of the Controller of Her Britannic Majesty's Stationery Office.

\section{References}

1 F. G. Celii and J. E. Butler, Annu. Rev. Phys. Chem., 42 (1991) 643.

2 A. H. Lettington and J. W. Steeds, (eds.), Philos. Trans. R. Soc. London, Ser. A, 342 (1993) 193.

3 P. K. Bachmann, D. Leers and D. U. Wiechert, Ber. Bunsenges. Phys. Chem., 95 (1991) 1390.

4 J. C. Angus, Thin Solid Films, 216 (1992) 126.

5 P. W. May, C. A. Rego, R. M. Thomas, M. N. R. Ashfold, K. N. Rosser, P. G. Partridge and N. M. Everitt, Proc. 3rd Int. Symp. on Diamond Materials, Honolulu, HI, May 1993, Electrochemical Society, Pennington, NJ, 1993.

6 P. W. May, P. G. Partridge, C. A. Rego, R. M. Thomas, M. N. R. Ashfold, K. N. Rosser and N. M. Everitt, submitted to J. Mater. Sci. Lett.

7 T. Obata and S. Morimoto, Proc. SPIE-Diamond Opt. II, 1146 (1989) 208 .

8 P. M. Natishan, A. A. Morrish, J. Glesener and P. E. Pehrsson, Proc. 3rd Int. Symp. on Diamond Materials, Honolulu, HI, May 1993. Electrochemical Society, Pennington, NJ, 1993.

9 P. W. May, C. A. Rego and R. M. Thomas, in preparation.

10 Q. S. Chia, M.Sc. Thesis, University of Bristol, 1993.

11 S. S. Park and J. Y. Lee, J. Appl. Phys., 69 (1991) 2618.

12 P. G. Partridge, P. W. May and M. N. R. Ashfold, in preparation. 